\title{
In this galaxy far, far away
}

\author{
The Nobel Prize in Physics 2020 has been awarded to Roger Penrose for his work on black hole formation, and to \\ Andrea Ghez and Reinhard Genzel for their observation of a supermassive compact object at the Galactic Centre.
}

( espite estimates for the number of black holes in the Milky Way ranging from ten million to a billion, we are only just beginning to uncover their secrets. Recent years have seen astonishing advances in black-hole research, from the iconic image of the shadow of a black hole in the centre of the galaxy Messier 87 to the detection of the first gravitational wave signal from a binary black-hole merger by the LIGO Collaboration. The latter earned Rainer Weiss, Barry Barish and Kip Thorne the 2017 Nobel Prize in Physics. And now, this year's prize caps half a decade of achievements by honouring two key contributions to our current understanding of black holes.

The 2020 Nobel Prize in Physics has been awarded to Roger Penrose "for the discovery that black hole formation is a robust prediction of the general theory of relativity", and to Andrea Ghez and Reinhard Genzel "for the discovery of a supermassive compact object at the centre of our Galaxy".

The existence of supermassive black holes has been contemplated since the end of the eighteenth century when John Michell and Pierre-Simon Laplace estimated the radius of a star capable of trapping light in its gravitational pull. Although their calculations of the point of no return were carried out in the framework of Newtonian mechanics, the result coincided with the Schwarzschild radius.

Robert Oppenheimer and Hartland Snyder then put forward the idea that an astronomical body with a radius less than or equal to the Schwarzschild radius would undergo gravitational collapse towards a singularity. This advance was met with scepticism at first - primarily as their proposal hung on a controversial assumption of spherical symmetry. Roy Kerr's generalization of the Schwarzschild metric to rotating bodies weakened this requirement, permitting singularities under departures from spherical symmetries, but failed to dispense of symmetry requirements entirely. And so the stage was set for a young Roger Penrose.

Inspired by Kerr's solution, Penrose set out to study gravitational collapse without relying on the assumption of spherical

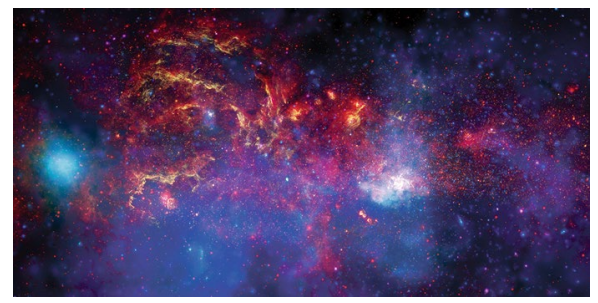

Credit: NASA / CXC / JPL-CALTECH / ESA-STSCI / Science Photo Library

symmetry. This required him to introduce the concept of trapped surfaces: whereas outward-directed light rays orthogonal to a spherical surface diverge, they converge for a trapped surface. Penrose showed that the existence of a trapped surface is independent of any symmetry assumptions, and that after its formation, gravitational collapse of a body with a positive energy density towards a singularity is inevitable within the framework of general relativity ${ }^{1}$.

The formation of (supermassive) black holes was thus put on solid footing almost 200 years after the works of Laplace and Michell, who had pondered how such objects might one day be observed. Because - as he wrote - no information was available from light, Michell suggested that the motions of other luminous bodies revolving around the black hole might allow one to infer its existence. If stars revolve around a supermassive black hole, their velocities should increase with decreasing distance, but the stars are expected to move faster with increasing distance around a spatially extended stellar mass cluster. By monitoring the stars orbiting the centre of our Galaxy, Andrea Ghez and Reinhard Genzel answered the question of whether a supermassive black hole exists with a resounding yes.

There are around ten million stars within one parsec of the Galactic Centre, so tracking the motion of a single star over time is challenging. The observations with telescopes at the European Southern Observatory in Chile and the Keck Observatory in Hawaii were carried out in the near-infrared and required long measurement times. And changes in the Earth's atmosphere blurred the signals, so Genzel's and Ghez's teams had to devise a clever technique to compensate for this effect.

By recording images with very short exposure times with an extremely sensitive detector and then stacking the images to account for spatial shifts, they achieved an image quality that was limited only by diffraction. Due to the short exposure times, the application of speckle imaging allowed them to monitor only the brightest stars. When adaptive optics became available in the early 2000s, the stars could be studied with spectrographs enabling measurements of both their compositions and radial velocities.

One star in particular piqued the researchers' interest: S2 travels around the Galactic Centre on a highly elliptical keplerian orbit with a surprisingly short period of around 16 years, whereas the same feat takes our Sun 200 million years. By tracking the motion of S2 over a full orbit and by measuring its radial velocities, the presence of a highly concentrated mass of around four million solar masses was inferred ${ }^{2,3}$ - excluding the possibility of a spatially extended stellar mass cluster.

The interpretation of this compact object as a supermassive black hole is further supported by near-infrared and X-ray flares observed in its vicinity, which might be caused by variations in the black hole's accretion flow. Together with the image of the black hole shadow in Messier 87, the discovery made by the teams around Genzel and Ghez provides the strongest observational evidence for the existence of supermassive black holes. As a next step, the Event Horizon Telescope plans to image the shadow of the supermassive black hole in the centre of our Galaxy — around 26,000 light years away.

Published online: 2 November 2020 https://doi.org/10.1038/s41567-020-01096-w

\footnotetext{
References

1. Penrose, R. Phys. Rev. Lett. 14, 57-59 (1965).

2. Schödel, R. et al. Nature 419, 694-696 (2002).

3. Ghez, A. et al. Astrophys. J. 586, L127-L131 (2003).
} 